\title{
ReaR
}

ISNN 19894090

Revista electrónica de AnestesiaR

Noviembre 2020

ARTÍCULOS DE REVISIÓN

\section{Análisis de la onda de presión arterial en Anestesiología y Cuidados Intensivos II.}

Paz Martín D.

Complejo Hospitalario de Toledo.

\section{Resumen}

Continuación de la serie de Análisis de la onda de presión arterial en Anestesiología y Cuidados Intensivos. En este artículo se profundiza en la calidad de la medición, un aspecto al que con frecuencia no se concede la importancia que tiene. La monitorización per se no mejorará los resultados clínicos del paciente o prevendrá complicaciones, sino las medidas que adoptemos basadas en la información que recibimos de esta monitorización. Por esto es imprescindible garantizar que la calidad de la monitorización sea óptima.

\section{Introducción}

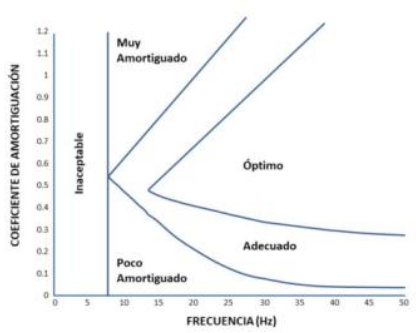

Continuación de la serie de Análisis de la onda de presión arterial en Anestesiología y Cuidados Intensivos. En este artículo se profundiza en la calidad de la medición, un aspecto al que con frecuencia no se concede la importancia que tiene.

La monitorización per se no mejorará los resultados clínicos del paciente o prevendrá complicaciones, sino las medidas que adoptemos basadas en la información que recibimos de esta monitorización. Por esto es imprescindible garantizar que la calidad de la monitorización sea óptima.

\section{Características de un sistema de medida de presión.}

La respuesta dinámica de los sistemas empleados en la monitorización de la presión arterial invasiva está determinada por su frecuencia resonante y su coeficiente de amortiguación (1).

Las fórmulas para calcular la frecuencia natural y el coeficiente de amortiguamiento son las siguientes (Fig. 1A y 1B):

$$
f_{n}=\frac{d}{8} \sqrt{\frac{3}{\pi L p V d}}
$$

Fig. 1A Fórmula de la frecuencia natural. 


$$
\varsigma=\frac{16 n}{d^{3}} \sqrt{\frac{3 L V d}{\pi p}}
$$

Fig. 1B Fórmula del Coeficiente de Amortiguamiento.

Donde:

$\mathrm{D}=$ diámetro del tubo;

$\mathrm{L}=$ longitud del tubo;

$\mathrm{p}=$ densidad del fluido;

$\mathrm{Vd}=$ desplazamiento del transductor por el volumen de fluido;

$\mathrm{n}=$ viscosidad del fluido.

\section{Frecuencia natural y resonancia}

Todo material cuando se golpea oscila a su frecuencia "natural" (Fn), que depende de propiedades físicas del material, como la densidad y el grosor, y también del material adyacente. Si se aplica una fuerza externa con una frecuencia similar a la frecuencia natural, el material oscilará en su amplitud máxima y esto se conoce como "resonancia".

Por lo tanto, si la frecuencia natural del sistema de monitorización de presión arterial invasiva está cerca de la frecuencia de cualquiera de los componentes de una onda arterial, vibrará en exceso y distorsionará la señal (reverberación). Para asegurar un registro exacto, la frecuencia natural de un sistema de monitorización debería ser al menos 6-10 veces más alta que la frecuencia más alta de la señal de entrada fundamental (frecuencia cardiaca).
Una onda de presión arterial se compone de una onda fundamental con una frecuencia de $1-3 \mathrm{~Hz}$ (frecuencia de $60-180 /$ minuto o $1-3$ por segundo) y una serie de ondas armónicas con frecuencias de hasta $25-30 \mathrm{~Hz}$.

La mayoría de los transductores de presión arterial disponibles comercialmente tienen una frecuencia natural de alrededor de $200 \mathrm{~Hz}$. Sin embargo, la incorporación de llaves de tres pasos y el empleo de tubos de mayor longitud pueden reducir la frecuencia natural del sistema con el resultado de un incremento de la presión arterial sistólica (PAS) y una disminución de la presión arterial diastólica (PAD).

\section{Coeficiente de amortiguamiento}

El amortiguamiento es la tendencia de factores como la fricción, las burbujas de aire y la complianza de los tubos, de absorber la energía y disminuir la amplitud de los picos y valles de la onda arterial. En un sistema arterial, todas las condiciones que afectarían negativamente al flujo de líquido (acodamientos, coágulos, burbujas de aire) aumentarían la amortiguación del mismo.

Todos estos dispositivos precisan cierto grado de amortiguamiento

(amortiguamiento crítico), pero si es excesivo o insuficiente, las lecturas de presión arterial se verán afectadas.

El coeficiente de amortiguamiento es una medida de la rapidez con la que un sistema oscilatorio vuelve a su etapa de reposo para detectar la siguiente onda. Un coeficiente de amortiguación bajo conduce a oscilaciones de gran amplitud y a lecturas erróneas de presión sistólica alta y baja presión diastólica. Por el contrario, un coeficiente de amortiguamiento alto causaría una disminución de la onda transmitida. 
Puede ser calculado mediante el test de lavado rápido (ver más adelante), midiendo la amplitud de 2 ondas consecutivas, dividiendo la menor entre la mayor y despejando el coeficiente de amortiguamiento en el nomograma de la figura 2.

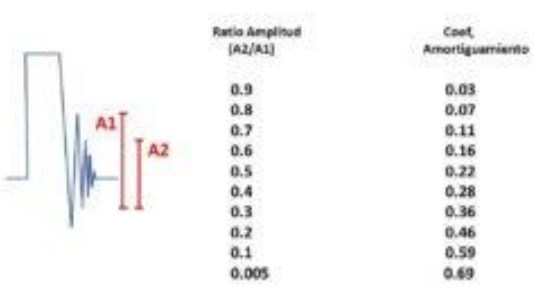

Fig. 2 Determinación del Coeficiente de Amortiguamiento.

Un coeficiente de 0,2-0,4 corresponde con un sistema con bajo amortiguamiento, entre $0,4-0,7$ un amortiguamiento óptimo, y entre 0,8 0,9 exceso de amortiguamiento.

El coeficiente de amortiguación óptimo de 0,7 proporciona el equilibrio entre respuesta rápida y precisión.

\section{Test de lavado de arrastre rápido o de onda cuadrada}

El amortiguamiento de un sistema de monitorización de presión puede evaluarse observando la respuesta del mismo a un test de lavado rápido. De esta forma se puede calcular la frecuencia natural y el coeficiente de amortiguación.

Al presionar el dispositivo de lavado del transductor se aplica un breve flujo a través del catéter arterial con una presión de $300 \mathrm{mmHg}$, que retorna inmediatamente a niveles basales cuando se deja de infundir el líquido. Esta maniobra muestra en el osciloscopio una onda cuadrada que alcanza una meseta de $300 \mathrm{mmHg}$. seguida de un descenso brusco. El sistema oscila antes de llegar a su estado de reposo mostrando una serie de ondas agudas que pueden ser empleadas para determinar la resonancia del sistema.

Diferentes tipos de curvas representan una amortiguación escasa, óptima y excesiva:

- Bajo coeficiente de amortiguación: el test de lavado producirá varias oscilaciones $(>3)$, amplificadas $(>1 / 3$ de la previa) por encima y debajo de la línea de base antes de regresar a esta línea. Este sistema presentará una resonancia excesiva y tenderá a sobrestimar la presión sistólica e infraestimar la presión diastólica (Fig. 3). Presión arterial media (PAM) sin cambios.

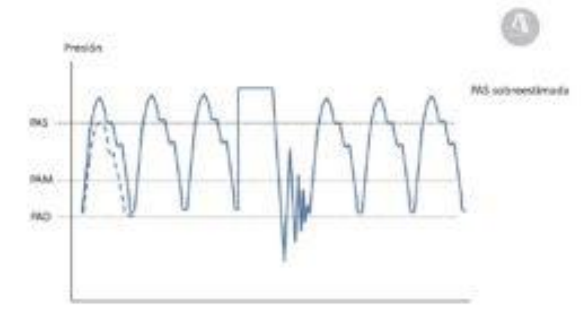

Fig. 3 Ondas arteriales no amortiguadas. PAS presión arterial sistólica; PAM presión arterial media; PAD presión arterial diastólica

- Sistema con amortiguación óptima se alcanzará la línea de base tras una oscilación (Fig. 4). La línea arterial adecuadamente amortiguada presentará las siguientes características:

1) El tiempo entre las oscilaciones será corto, habitualmente menor de 30 mseg., lo que corresponde a una frecuencia natural de $33 \mathrm{~Hz}$. 2) Habrá al menos una oscilación. 3) No debería haber más de 2 . 4) La amplitud de cada oscilación no debe ser mayor que un tercio de la amplitud de la oscilación previa. 
5) Las ondas arteriales deben mostrar una muesca dícrota clara. Este elemento se produce a partir de las ondas de reflexión de alta frecuencia que habitualmente son de baja amplitud y, por lo tanto, más susceptibles a la amortiguación del sistema.

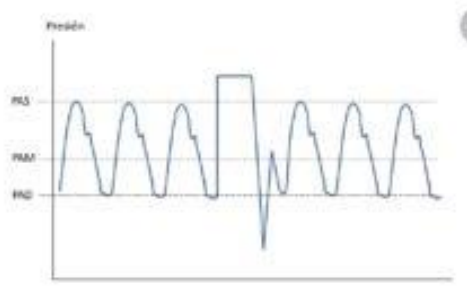

Fig. 4 Sistema con amortiguación óptima. PAS presión arterial sistólica; PAM presión arterial media; PAD presión arterial diastólica

- Amortiguación excesiva: se volverá a la línea de base sin oscilaciones. El sistema infraestimará la PAS, sobrestimará la PAD pero la PAM no se verá modificada (Fig. 5). Además, se observa una pérdida de la muesca dícrota, resultado de la amortiguación de los elementos de alta frecuencia que dejan de contribuir en la forma de la onda de pulso arterial.

Para corregir esta situación se debe:

- Evaluar la presencia de coágulos y/o burbujas de aire en el catéter o en la tubuladura.

- Verificar que la punta del catéter arterial no haga pared con el vaso arterial.

- Identificar y corregir cualquier acodamiento del catéter o de la tubuladura.

- Cambiar la tubuladura a una de longitud más corta.

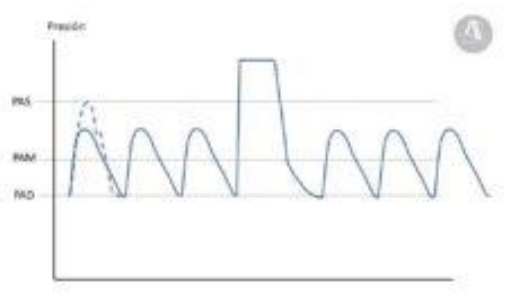

Fig. 5 Sistema con amortiguación excesiva. PAS presión arterial sistólica; PAM presión arterial media; PAD presión arterial diastólica

\section{Determinantes de la frecuencia natural}

La Fn es el tiempo entre oscilaciones. Debería ser del orden de $30 \mathrm{~Hz}$ (30 veces por segundo) para monitorizar de forma adecuada frecuencias cardiacas en torno a $180 \mathrm{lpm}(3 \mathrm{~Hz})$. Se puede calcular dividiendo la velocidad de la pantalla por la longitud de onda de las ondas resonantes. Por lo tanto, cuanto más cerca estén los ciclos de oscilación, mayor será la frecuencia natural.

Imprimiendo en un trazado de papel el test de lavado se puede calcular la Fn mediante la siguiente fórmula (Fig. 6):

$\mathrm{Fn}=$ velocidad del trazado $(\mathrm{mm} / \mathrm{s}) /$ longitud de onda de las ondas resonantes (longitud en $\mathrm{mm}$ de un ciclo)

Ej. Velocidad de pantalla $=25 \mathrm{~mm} / \mathrm{s}$; longitud de onda $=1 \mathrm{~mm} ; \mathrm{Fn}=25 / 1=$ $25 \mathrm{~Hz}$

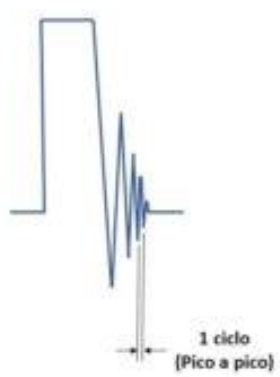

Fig. 6 Determinación de la longitud de onda de las ondas resonantes.

¿Cuándo realizar un test de lavado rápido? 
- Cada vez que la curva parezca amortiguada en exceso.

- Cada vez que los cambios fisiológicos del paciente aumenten la demanda del sistema de monitorización (ej. aumento de la frecuencia cardiaca, vasoconstricción).

- Tras la apertura del sistema.

- Antes de implementar terapias hemodinámicas o de cambiarlas.

- Cuando existan dudas de la exactitud de las mediciones.

- Al menos cada 8 horas.

\section{Significación clínica}

Hay una dependencia del coeficiente de amortiguamiento óptimo de la frecuencia natural (2). Cada componente del sistema de monitorización tiene su propia Fn y Coeficiente de Amortiguamiento.

Aunque los requisitos técnicos deseados para la monitorización de la presión arterial son una frecuencia natural superior a $25 \mathrm{~Hz}$ y un coeficiente de amortiguación de 0,7 , estas condiciones rara vez se cumplen en la práctica clínica habitual. La mayoría de los sistemas de transductores de tubos de catéter están amortiguados (coeficiente de amortiguación de 0,15 a 0,45) y tienen una frecuencia natural aceptable de 12-25 Hz, especialmente si la frecuencia cardíaca es inferior a $90 /$ min o 1,5 Hz. Con Fn del sistema muy bajas (inferior a 7Hz), no hay coeficiente de amortiguamiento que resulte aceptable. Sin embargo, conforme la Fn del sistema aumenta, un rango más ancho de coeficiente de amortiguamiento resulta aceptable porque la amortiguación tendrá un efecto mínimo en el registro de la onda arterial. La Figura 7 muestra la relación entre el coeficiente de amortiguamiento y la Fn de los sistemas de monitorización de la presión arterial (3). Hay cinco situaciones posibles.
1. Adecuado: registro preciso de la mayoría de las formas de onda de presión que se observan en la clínica habitual.

2. Muy amortiguado.

3. Defecto de amortiguación.

4. Inaceptable - frecuencia natural $<7 \mathrm{~Hz}$. Obsérvese que con estas Fn, no hay coeficiente de amortiguación que resulte aceptable.

\section{5. Óptimo.}

Si la frecuencia natural es baja $(10 \mathrm{~Hz})$, el coeficiente de amortiguación debe estar entre $0,45-0,6$ o el sistema resonaría y registraría una presión de pulso erróneamente amplia.

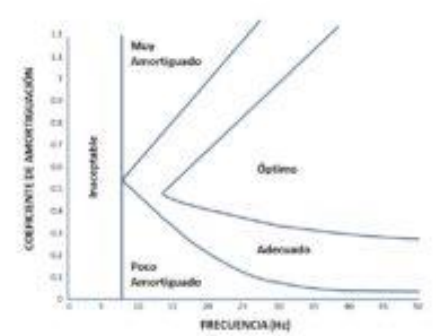

Fig. 7 Relación amortiguamiento y frecuencia natural.

\section{Conclusión}

La respuesta dinámica de los sistemas empleados en la monitorización de la presión arterial invasiva está determinada por su frecuencia resonante y su coeficiente de amortiguación. Aunque hay descritas fórmulas más o menos complejas, estas variables pueden ser evaluadas de forma sencilla clínicamente mediante un test de lavado rápido.

\section{Bibliografía}

1. Kleinman B. Understanding natural frequency and damping and how they relate to the measurement of blood pressure. J Clin Monit. 1989;5:137-47. ( 
2. Gardner RM. Direct blood pressure measurement - dynamic responds requirements. Anesthesiology. 1981;54:227-36. (ubMed)

3. Rogers P. Invasive Hemodynamic Monitoring. En: Pinsky MR, editor. Applied Cardiovascular Physiology. Springer; 1997. p 113-126. (PMC)

Cómo citar: Paz-Martín, D. (2020). Análisis de la onda de presión arterial en Anestesiología y Cuidados Intensivos I. Revista Electrónica AnestesiaR, 12(6),

4. https://doi.org/10.30445/rear.v12i6.858
Correspondencia al autor

Daniel Paz Martín

dpaz@anestesiar.org

MD PhD EDAIC EDIC Servicio Anestesiología y Reanimación.

Complejo Hospitalario de Toledo. Sección de Cuidados Intensivos de la SEDAR

Aceptado para el blog en abril de 2020 